\title{
Dot Product Representations of Planar Graphs
}

\author{
Ross J. Kang ${ }^{1, \star}$ and Tobias Müller ${ }^{2, \star \star}$ \\ 1 Durham University \\ ross .kang@gmail.com \\ 2 Centrum Wiskunde \& Informatica \\ tobias@cwi.nl
}

\begin{abstract}
A graph $G$ on $n$ vertices is a $k$-dot product graph if there are vectors $u_{1}, \ldots, u_{n} \in \mathbb{R}^{k}$, one for each vertex of $G$, such that $u_{i}^{T} u_{j} \geq 1$ if and only if $i j \in E(G)$. Fiduccia, Scheinerman, Trenk and Zito (1998) asked whether every planar graph is a 3-dot product graph. We show that the answer is "no". On the other hand, every planar graph is a 4-dot product graph.
\end{abstract}

\section{Introduction and Statement of Results}

We study a type of geometric representation of graphs using vectors from $\mathbb{R}^{k}$ for some $k \in \mathbb{N}$. Let $G$ be a graph with $n$ vertices. We say $G$ is a $k$-dot product graph if there exist vectors $u_{1}, \ldots, u_{n} \in \mathbb{R}^{k}$ such that $u_{i}^{T} u_{j} \geq 1$ if and only if $i j \in E(G)$. An explicit set of vectors in $\mathbb{R}^{k}$ that exhibits $G$ in this way is called a $k$-dot product representation of $G$. The dot product dimension of $G$ is the least $k$ such that there is a $k$-dot product representation of $G$. (Notice that $G$ can be trivially represented in $\mathbb{R}^{|E(G)|}$, so that the dot product dimension is finite for all graphs.)

The well-studied class of threshold graphs coincides with the 1-dot product graphs; consult the monograph by Mahadev and Peled [6] for a comprehensive survey of results on these and related structures.

Partially motivated by the striking application by Lovász of a similar geometric representation to an important problem on Shannon capacity [5], Reiterman, Rödl and Šiňajová [789] studied the dot product dimension extensively and obtained several bounds in terms of threshold dimension, sphericity, chromatic number, maximum degree, maximum average degree, and maximum complementary degree; they also detailed various examples. Arriving from a different direction Fiduccia, Scheinerman, Trenk and Zito [3] also considered dot product dimension and, for example, analysed bipartite, complete multipartite, and interval graphs. Both Reiterman et al. and Fiduccia et al. proved that every forest is a 3 -dot product graph $8 \mid 3$.

\footnotetext{
* Research partially supported by the Engineering and Physical Sciences Research Council (EPSRC), grant EP/G066604/1.

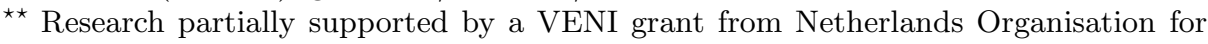
Scientific Research (NWO).
} 
Seeing a potential extension to this result, Fiduccia et al. asked whether every planar graph is a 3-dot product graph. Here we will answer this in the negative by describing a counterexample. In contrast, we show that any planar graph has dimension at most 4 .

Theorem 1. Every planar graph is a 4-dot product graph, and there exist planar graphs which are not 3-dot product graphs.

It remains open to characterise which planar graphs have dot product dimension exactly 4 .

The structure of the paper is as follows. In the next section, we develop some notation and review some spherical geometry. In Section 3, we present our counterexample. We show how every planar graph has a 4-dot product representation in Section 4 .

\section{Preliminaries}

For $u, v \in S^{2}$, let us denote by $[u, v]$ the (shortest) spherical arc between $u$ and $v$. Let $\operatorname{dist}_{S^{2}}(u, v)$ denote the length of $[u, v]$. Then one can see that $\operatorname{dist}_{S^{2}}(u, v)$ equals the angle between the two vectors $u, v \in S^{2}$. It can thus be expressed as

$$
\operatorname{dist}_{S^{2}}(u, v)=\arccos \left(v^{T} u\right) .
$$

For $r \geq 0$, let the spherical cap of radius $r$ around $v \in S^{2}$ be defined as

$$
\operatorname{cap}(v, r):=\left\{u \in S^{2}: \operatorname{dist}_{S^{2}}(u, v) \leq r\right\} .
$$

Suppose that $u, v, w \in S^{2}$ are three points on the sphere in general position. We shall call the union of the three circular arcs $[u, v],[v, w],[u, w]$ a spherical triangle. Similarly one can define a spherical polygon.

Let us write $a:=\operatorname{dist}_{S^{2}}(u, v), b:=\operatorname{dist}_{S^{2}}(u, w), c:=\operatorname{dist}_{S^{2}}(v, w)$, and let $\gamma$ denote the angle between $[u, v]$ and $[u, w]$. See Figure 1 .

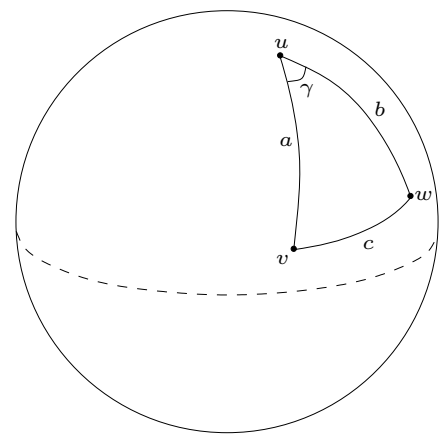

Fig. 1. A spherical triangle 
Recall the spherical law of cosines:

$$
\cos (c)=\cos (a) \cos (b)+\sin (a) \sin (b) \cos (\gamma) .
$$

The spherical law of cosines can be rephrased as:

$$
v^{T} w=\left(u^{T} v\right) \cdot\left(u^{T} w\right)+\cos (\gamma) \sqrt{\left(1-\left(u^{T} v\right)^{2}\right)\left(1-\left(u^{T} w\right)^{2}\right)} .
$$

This second form will be more useful for our purposes.

\section{Planar Graphs That Are Not 3-Dot Product Graphs}

We will construct graphs $F, G, H$ as follows.

(i) We start with $K_{4}$, the complete graph on the four vertices $t_{1}, t_{2}, t_{3}, t_{4}$.

(ii) To obtain $F$, we replace each edge $t_{i} t_{j}$ of $K_{4}$ by a path $t_{i} t_{i j} t_{j i} t_{j}$ of length 3.

(iii) An embedding of the graph $F$ divides the plane into four faces. To obtain $G$, we place an additional vertex inside each face of $F$ and connect it to all vertices on the outer cycle of the face. Here, $f_{i}$ will denote the vertex in the face whose limiting cycle does not contain $t_{i}$.

(iv) Finally, to obtain $H$ we attach four leaves to each vertex of $G$.

The graphs $G$ and $H$ are depicted in Figure 2, For $k \in \mathbb{N}$, let the graph $H_{k}$ consist of $k$ disjoint copies of $H$. Clearly $H_{k}$ is planar for all $k$. In the rest of this section, we shall prove the following.

Theorem 2. The graph $H_{k}$ is not a 3-dot product graph, for sufficiently large $k$.
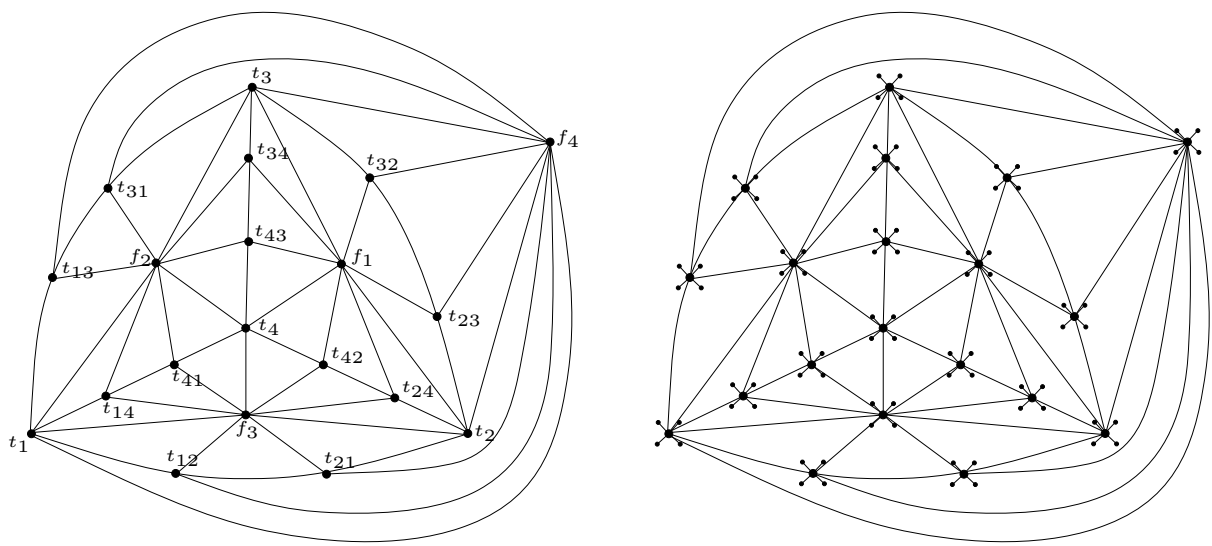

Fig. 2. The graphs $G, H$ 
Proof of Theorem 2. The proof is by contradiction. Let us assume that every $H_{k}$ has a 3 -dot product representation. The proof is divided into a number of intermediate steps. The details of the proofs of Claims 3 through 8 below can be found in the journal version of this paper.

Claim 3. For every $\eta>0$ there is a 3 -dot product representation of $H$, with $\|u(t)\|<1+\eta$ for all $t \in V(H)$.

Let us fix a small $\eta$ (say $\eta:=10^{-10}$ ), and let $u: V(H) \rightarrow \mathbb{R}^{3}$ be the representation provided by Claim [3. For $s \in V(H)$ let us write $l(s):=\|u(s)\|, v(s):=$ $u(s) /\|u(s)\|$. Let us observe that

$$
s t \in E(H) \quad \text { if and only if } \quad v(s)^{T} v(t) \geq 1 / l(s) l(t) .
$$

Recall that $G \subseteq H$ is the subgraph induced by all non-leaf vertices.

Claim 4. For every $s \in V(G)$ we have $l(s)>1$.

Claim 5. Suppose that $s t, s^{\prime} t^{\prime} \in E(G)$ are edges with $s, s^{\prime}, t, t^{\prime}$ distinct and suppose that the $\operatorname{arcs}[v(s), v(t)]$ and $\left[v\left(s^{\prime}\right), v\left(t^{\prime}\right)\right]$ cross. Then at least one of $s s^{\prime}, s t^{\prime}, t s^{\prime}, t t^{\prime}$ is also an edge of $G$.

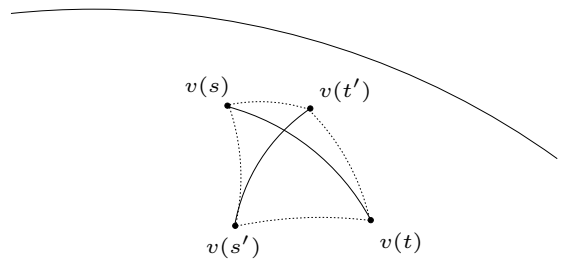

Claim 6. Suppose that $s_{1}, s_{2}, s_{3}$ form a clique in $G$, and $v(s)$ lies inside the (smaller of the two areas defined by the) spherical triangle defined by $v\left(s_{1}\right)$, $v\left(s_{2}\right), v\left(s_{3}\right)$. Then either $s_{1} s \in E(G)$ or $s_{2} s \in E(G)$ or $s_{3} s \in E(G)$.

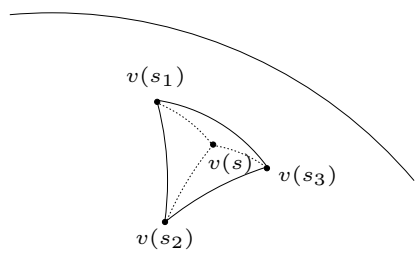

From now on, let us write $v_{i}:=v\left(t_{i}\right), l_{i}=l\left(t_{i}\right)$ and $v_{i j}=v\left(t_{i j}\right), l_{i j}=$ $l\left(t_{i j}\right)$. By Claim 5, the arcs $\left[v_{i j}, v_{j i}\right]$ and $\left[v_{k l}, v_{l k}\right]$ do not cross each other (for $\{i, j\} \neq\{k, l\})$. However, the arc $\left[v_{i j}, v_{j i}\right]$ could cross an arc of the form $\left[v_{i}, v_{i k}\right]$ or $\left[v_{j}, v_{j k}\right]$.

Let $C$ denote the cycle $t_{1} t_{12} t_{21} t_{2} t_{23} t_{32} t_{3} t_{31} t_{13} t_{1}$ in $G$, and let $P$ denote the corresponding spherical polygon. Because each circular arc corresponding to an edge has length at most $\rho$, we have $P \subseteq \operatorname{cap}\left(v_{1}, 5 \rho\right)$. Also note that $S^{2} \backslash P$ consists of at least two path-connected components (by the Jordan Curve Theorem). As 
$\rho$ is small, exactly one of these components has area $>3.9 \pi$. We shall refer to this component as the "outside" of $P$, and the union of the other components we will call the "inside".

Claim 7. We can assume without loss of generality that $v_{4}$ lies inside the polygon $P$.

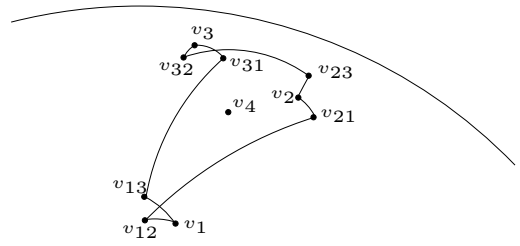

Claim 8. $v_{4}$ lies inside the spherical triangle defined by $v\left(f_{4}\right)$ and two consecutive points on $P$.

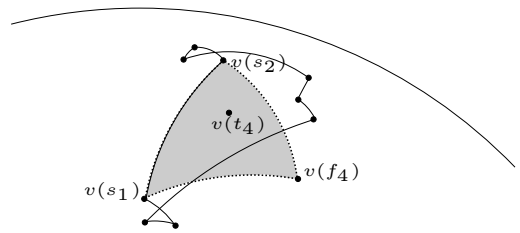

Since $f_{4}, s_{1}, s_{2}$ form a triangle in $G$, Claim 6 implies that $t_{4}$ must be adjacent to at least one of them. This is a contradiction, since $t_{4}$ is neither adjacent to $f_{4}$ nor to any vertex of $C$. This completes the proof of Theorem 2 .

\section{All Planar Graphs Are 4-Dot Product Graphs}

The Colin de Verdière parameter $\mu(G)$ of a graph $G$ is the maximum co-rank over all matrices $M$ that satisfy

(i) $M_{i j}<0$ if $i j \in E(G)$;

(ii) $M_{i j}=0$ if $i j \notin E(G)$ and $i \neq j$;

(iii) $M$ has exactly one negative eigenvalue;

(iv) if $X$ is symmetric with $X_{i j}=0$ for all $i j \in E(G)$ and $X_{i i}=0$, for all $i$, and $M X=0$, then we must have $X=0$.

This parameter was introduced Y. Colin de Verdiére in [12], where it is shown that planar graphs are exactly the graphs $G$ with $\mu(G) \leq 3$.

Kotlov, Lovasz and Vempala [4] introduced the following related parameter. Let $\nu(G)$ denote the smallest $d$ such that there exist vectors $u_{1}, \ldots, u_{n} \in \mathbb{R}^{d}$ that satisfy

(i) $u_{i}^{T} u_{j}=1$ if $i j \in E(G)$;

(ii) $u_{i}^{T} u_{j}<1$ if $i j \notin E(G)$ and $i \neq j$; 
(iii) if $X$ is a symmetric $n \times n$ matrix such that $X_{i j}=0$ for all $i j \in E(G)$ and $X_{i i}=0$ for all $i$, and $\sum_{j} X_{i j} u_{j}=0$ for all $i$, then $X=0$.

Clearly, every graph $G$ is a $\nu(G)$-dot product graph. However, because (i) asks for equality and because of the extra demand (iii) $G$ might also be a $k$-dot product graph for some $k<\nu(G)$. The relation between $\nu(G)$ and $\mu(G)$ is given by the following result.

Theorem 9 ([4]). If $G \neq K_{2}$ then $\nu(G)=n-1-\mu(\bar{G})$.

That $K_{2}$ is a 4-dot product graph is obvious. That every other planar graph is a 4-dot product graph is a direct consequence of Theorem 9 and the following result.

Theorem 10 ([4]). If $G$ is the complement of a planar graph then $\mu(G) \geq n-5$.

\section{References}

1. Colin de Verdière, Y.: Sur un nouvel invariant des graphes et un critère de planarité. J. Combin. Theory Ser. B 50(1), 11-21 (1990)

2. de Verdière, Y.C.: On a new graph invariant and a criterion for planarity. In: Graph structure theory, Contemp. Math. Amer. Math. Soc., Seattle, WA, vol. 147, pp. 137-147. RI, Providence (1991)

3. Fiduccia, C.M., Scheinerman, E.R., Trenk, A., Zito, J.S.: Dot product representations of graphs. Discrete Math. 181(1-3), 113-138 (1998)

4. Kotlov, A., Lovász, L., Vempala, S.: The Colin de Verdière number and sphere representations of a graph. Combinatorica 17(4), 483-521 (1997)

5. Lovász, L.: On the Shannon capacity of a graph. IEEE Trans. Inform. Theory 25(1), 1-7 (1979)

6. Mahadev, N.V.R., Peled, U.N.: Threshold graphs and related topics. Annals of Discrete Mathematics, vol. 56, North-Holland Publishing Co, Amsterdam (1995)

7. Reiterman, J., Rödl, V., Šiňajová, E.: Embeddings of graphs in Euclidean spaces. Discrete Comput. Geom. 4(4), 349-364 (1989)

8. Reiterman, J., Rödl, V., Šiňajová, E.: Geometrical embeddings of graphs. Discrete Math. 74(3), 291-319 (1989)

9. Reiterman, J., Rödl, V., Šiňajová, E.: On embedding of graphs into Euclidean spaces of small dimension. J. Combin. Theory Ser. B 56(1), 1-8 (1992) 